\title{
ROLE OF COLOUR DOPPLER IN EVALUATION OF ACUTE DEEP VEIN THROMBOSIS OF LOWER LIMB
}

\author{
Vijay Bahadur Singh ${ }^{1}$, Punya Pratap Singh ${ }^{2}$, Rajesh Malik³, Lovely Kaushal4, Vijay Verma ${ }^{5}$, Abhijeet Patil6, Anuja Patil ${ }^{7}$
}

${ }_{1}^{1}$ Assistant Professor, Department of Radiodiagnosis, Ratlam Medical College, Ratlam, Madhya Pradesh, India.

${ }^{2}$ Associate Professor, Department of Radiodiagnosis, Bundelkhand Medical College, Sagar, Madhya Pradesh, India.

3 Professor, Department of Radiodiagnosis, AIIMS, Bhopal, Madhya Pradesh, India.

${ }^{4}$ Professor, Department of Radiodiagnosis, Gandhi Medical College, Bhopal, Madhya Pradesh, India.

5Professor, Department of Radiodiagnosis, Gandhi Medical College, Bhopal, Madhya Pradesh, India.

${ }^{6}$ Professor, Department of Radiodiagnosis, Vidisha Medical College, Vidisha, Madhya Pradesh, India.

${ }^{7}$ Consultant Radiologist, Department of Radiodiagnosis, Vision Diagnostic Centre, Ratlam, Madhya Pradesh, India.

\section{BACKGROUND}

\section{ABSTRACT}

Acute deep vein thrombosis (DVT) is a well-recognized contributor to increased morbidity and mortality following trauma and elective musculoskeletal procedures. While ascending venography is the gold standard for the diagnosis of acute DVT, various noninvasive surveillance modalities including colour Doppler have been introduced. With the addition of colour flow, Doppler ultrasound allows improved identification of vessels by visualizing blood flow and establishes the direction of flow.

\section{MATERIALS AND METHODS}

The study was carried out at Department of Radio Diagnosis, G.M.C., Bhopal in 100 acute DVT patients from 1/7/08 to 30/11/09. The study was carried out with GE logic 2 expert by using linear transducer of 7.5 to $10 \mathrm{MHz}$. Scan starting from iliac segment then cephalad up to iliac bifurcation and caudally up to tibial veins.

\section{RESULTS}

In 100 acute DVT patients, in about 94\% of symptomatic patients, thrombus was seen involving veins above knee. Probe compressibility was absent in $98 \%$ patients. Colour flow was absent in $100 \%$ patients.

\section{CONCLUSION}

Colour Doppler is better predictor of acute DVT because it is more sensitive \& specific, non-invasive, painless, widely available, easy to use, less expensive \& has no radiations.

\section{KEY WORDS}

Acute, Colour Doppler, Deep Vein Thrombosis, Augmentation.

HOW TO CITE THIS ARTICLE: Singh VB, Singh PP, Malik R, et al. Role of colour Doppler in evaluation of acute deep vein thrombosis of lower limb. J. Evolution Med. Dent. Sci. 2019;8(08): 512-516, DOI: 10.14260/jemds/2019/113

\section{BACKGROUND}

Acute deep vein thrombosis (DVT) is a well-recognized contributor to increased morbidity and mortality following trauma and elective musculoskeletal procedures.

Due to the inaccuracy of the bedside clinical diagnosis of acute DVT various invasive and non-invasive surveillance modalities have been developed. Ultrasound has recently gained popularity for use in the diagnosis of symptomatic acute DVT due to its low morbidity and non-invasive nature.

While ascending venography remains the gold standard for the diagnosis of acute DVT, various non-invasive surveillance modalities have been introduced. These include [1125] labelled fibrinogen, impedance plethysmography, magnetic resonance venography, and ultrasonography.

Ultrasound utilizes the reflections of sound waves to evaluate soft tissue structures.

'Financial or Other Competing Interest': None.

Submission 26-12-2018, Peer Review 09-02-2019,

Acceptance 16-02-2019, Published 25-02-2019.

Corresponding Author:

Dr. Punya Pratap Singh,

Flat No. 9, 3' ${ }^{\text {rd }}$ Floor, Type III Quarters,

Block-B, BMC Residential Complex,

Madhya Pradesh-470001,

India.

E-mail: drpunya@gmail.com

DOI: $10.14260 /$ jemds/2019/113

\section{(c) (i) $(9)$}

Two modes of ultrasound are used in the evaluation of acute DVT: B-mode and duplex ultrasound. This enables the visualization of venous anatomy without the use of contrast media.

More recently, duplex scanning has been introduced as another modality for acute DVT diagnosis. Duplex ultrasound combines B-mode imaging with pulsed-wave Doppler technology. Doppler ultrasonography is based on the physical principles of Christian Doppler (1803-1853), who described changes in sound frequency when reflected from a moving object. Pulsed-wave Doppler can be utilized to evaluate the direction and pulsatile nature of blood flow within vessels. With the addition of colour flow, Doppler ultrasound allows improved identification of vessels by visualizing blood flow and establish the direction of flow to differentiate arteries from veins. However, its utility to enhance the identification of acute DVT in the clinical setting is still debated.

No single physical finding or combination of signs and symptoms is sufficiently accurate to establish the diagnosis of acute deep vein thrombosis.

The clinical diagnosis using individual tests such as the Homans sign is fraught with error. $75-84 \%$ of post-operative patients suspected of having proximal acute deep vein thrombosis on basis on classical signs and risk factors are found negative when formal diagnostic testing is completed.

Acute thrombosis refers to a period of 14 days during which the vein is inflamed. Within this period thrombus can 
embolize to pulmonary circulation causing clinically significant or life-threatening pulmonary embolism.

\section{Aims and Objectives}

1. To study the distribution of thrombus in acute lower extremity deep vein thrombosis.

2. Study to assess the factors prone to give rise to acute DVT.

\section{MATERIALS AND METHODS}

The observational study was carried out at Department of Radio diagnosis in 100 acute DVT patients from 1/7/08 to $30 / 11 / 09$. The study was carried out by USG machine of GE logic 2 expert scan by using linear transducer of $7.5 \mathrm{MHz}$ to $10 \mathrm{MHz}$.

\section{Criteria for Selection of Patients}

All patients having acute deep venous thrombosis.

\section{Criteria for Exclusion of Patients}

All patients having deep venous thrombosis for more than 14 days (Correlated with previous scan / other report showing diagnosis of deep vein thrombosis).

\section{MATERIAL}

1. 100 acute deep venous thrombosis patients referred from orthopaedic, medicine, general surgery, obstetrics and gynaecology, radiotherapy and other departments.

2. USG machine of GE logic 3 expert scan and linear transducer.

3. Comfortable bed for patients.

4. Jelly.

Technique for Lower Extremity Venous Ultrasound Examination

\section{Patient Position}

Clear visualization of lower extremity veins requires adequate distension of venous system. To this end the lower extremity must be dependent which may be accomplished by steeply elevating the head of the examination table or by examining the patient in the sitting position. The patient is examining room should be sufficiently warm to prevent vasoconstriction, which results in poor venous distension.

\section{Step 1}

The iliac segment identifies the external iliac vein at the groin and follow it cephalad with long axis images. Locate the iliac bifurcation or its approximate position. Follow the common iliac vein cephalad to the inferior vena cava.

\section{Step 2}

The femoral segment uses long axis images to identify the external iliac vein at the groin and follow it distally into the common femoral vein. Note the entrance of great saphenous vein. Check Doppler characteristics at the common femoral level. Identify the deep femoral vein and confirm its patency.

\section{Step 3}

The great saphenous vein confirm that the proximal portion of the great saphenous vein is patent with long axis colour flow veins. Examine as much of the vein as is clinically indicated using short axis intermittent compression.

\section{Step 4}

The popliteal segment using long axis veins, locate distal portion of the superficial femoral vein as high as possible in the adductor canal. Follow the superficial femoral vein distal into the popliteal segment to the junction of the tibial trunks confirm the compressibility of the popliteal vein and the tibial trunks with short axis views and intermittent compression watch for popliteal vein duplication.

\section{Step 5}

The calf vein examining the posterior tibial veins in their entirety starting either at the popliteal space or at the ankle. Examine the anterior tibial veins \& peroneal veins with long axis colour flow images. Examine the gastrocnemius and soleal veins as clinically indicated, using long or short axis veins.

\section{RESULTS}

\section{Age-Wise Distribution of Cases}

First, we distribute the hundred acute deep venous thrombosis patients in seven age groups according to their age.

\begin{tabular}{|c|c|c|c|}
\hline Sl. No. & Age Group (Yrs.) & No. of Patients & Percentage \\
\hline 1 & $0-9$ & 0 & 0 \\
\hline 2 & $10-19$ & 3 & 3 \\
\hline 3 & $20-29$ & 13 & 13 \\
\hline 4 & $30-39$ & 24 & 24 \\
\hline 5 & $40-49$ & 27 & 27 \\
\hline 6 & $50-59$ & 17 & 17 \\
\hline 7 & $>60$ & 16 & 16 \\
\hline \multicolumn{2}{|r|}{ Total } & 100 & \\
\hline
\end{tabular}

Majority of patients [60\%] in our study were present in age group 40 yrs. and above.

In our study, majority of the males were of the age group 30-50 yrs.

Majority of female patients were of age group 40-60 yrs.

\begin{tabular}{|c|c|c|c|}
\hline Sl. No. & Diagnosis & No. of Patients & \% \\
\hline 1 & Post Trauma & 17 & 17 \\
\hline 2 & Post-Surgical & 16 & 16 \\
\hline 3 & Puerperal & 6 & 6 \\
\hline 4 & $\begin{array}{c}\text { Neurological Patients (CVA, } \\
\text { TBM \& Cauda Equina } \\
\text { Syndrome) }\end{array}$ & 15 & 15 \\
\hline 5 & Cancer Patients & 6 & 6 \\
\hline 6 & Cardiac Patients (MI \& CCF) & 7 & 7 \\
\hline 7 & Varicose Veins & 4 & 4 \\
\hline 8 & Insect Bite & 6 & 6 \\
\hline 9 & Idiopathic & 23 & 23 \\
\hline \multicolumn{2}{|c|}{ Total } & $\mathbf{1 0 0}$ & \\
\hline Table 2. Distribution of Patients as Per Cause of Acute DVT \\
\hline \multicolumn{2}{|c|}{}
\end{tabular}

Majority of patients in our study (33\%) were posttraumatic and post-surgical. 


\begin{tabular}{|c|c|c|c|c|}
\hline \multirow{3}{*}{$\begin{array}{c}\text { Segment } \\
\text { Inferior Vena Cava } \\
\end{array}$} & \multicolumn{2}{|c|}{$\begin{array}{c}\text { Unilateral } \\
\text { Limb }\end{array}$} & $\begin{array}{c}\text { Both } \\
\text { limbs }\end{array}$ & \multirow{2}{*}{$\frac{\text { Total }}{6}$} \\
\hline & \multicolumn{3}{|c|}{6} & \\
\hline & Right & Left & & \\
\hline Common Iliac Vein & 3 & 1 & 2 & 4 \\
\hline External Iliac Vein & 8 & 6 & 4 & 14 \\
\hline Common Femoral Vein & 28 & 24 & 8 & 54 \\
\hline Superficial Femoral Vein & 43 & 41 & 9 & 84 \\
\hline Deep Femoral Vein & 14 & 10 & 5 & 24 \\
\hline Popliteal Vein & 36 & 38 & 4 & 74 \\
\hline Anterior Tibial Vein & 12 & 11 & 1 & 23 \\
\hline Posterior Tibial Vein & 24 & 20 & 2 & 44 \\
\hline Peroneal Vein & 5 & 3 & 1 & 8 \\
\hline Great Saphenous Vein & 8 & 2 & 0 & 10 \\
\hline Short Saphenous Vein & 2 & 2 & 0 & 4 \\
\hline
\end{tabular}

Large thrombi involving many venous systems were more commonly seen in our study and the involvement of proximal deep veins was noted much more commonly than that of the distal veins. Bilateral disease was more commonly seen in proximal veins and the unilateral involvement was more common in distal veins.

\section{Distribution According to B-Mode Sonographic Findings}

All 100 patients were subjected to B-mode sonography and scanned on A) lumen diameter B) morphology of thrombus C) compressibility and D) collateral veins.

\section{A) Lumen Diameter}

\begin{tabular}{|c|c|c|c|}
\hline Sl. No. & Lumen Diameter & No. of Patients & $\%$ \\
\hline 1 & Increased & 97 & 97 \\
\hline 2 & Decreased & 2 & 2 \\
\hline 3 & Normal & 1 & 1 \\
\hline \multicolumn{2}{|r|}{ Total } & 100 & 100 \\
\hline \multicolumn{4}{|c|}{ Table 4. Lumen Diameter } \\
\hline
\end{tabular}

In acute DVT, lumen diameter was increased in $97 \%$ of patients in our study.

\section{B) Thrombus Morphology}

\begin{tabular}{|c|c|c|c|}
\hline Sl. No. & Thrombus Morphology & No. of Patients & $\%$ \\
\hline 1 & Completely Anechoic & 55 & 55 \\
\hline 2 & Anechoic to Isoechoic & 45 & 45 \\
\hline \multicolumn{4}{|c|}{ Table 5. Thrombus Morphology } \\
\hline
\end{tabular}

In our study, we found that $55 \%$ of patients had completely anechoic thrombus whereas rest of them had anechoic to isoechoic thrombus.

C) Compressibility

\begin{tabular}{|c|c|c|c|}
\hline Sl. No. & Compressibility & No. of Patients & $\%$ \\
\hline 1 & Absent & 98 & 98 \\
\hline 2 & Present & 2 & 2 \\
\hline \multicolumn{2}{|r|}{ Total } & 100 & 100 \\
\hline
\end{tabular}

In our study, we found that only $2 \%$ of thrombosed were compressible despite absence of colour flow and increased lumen diameter.

\section{D) Collateral Veins}

The patients were distributed on the basis of presence or absence of collateral veins. In our study, only $3 \%$ of patients had detectable collateral flow.

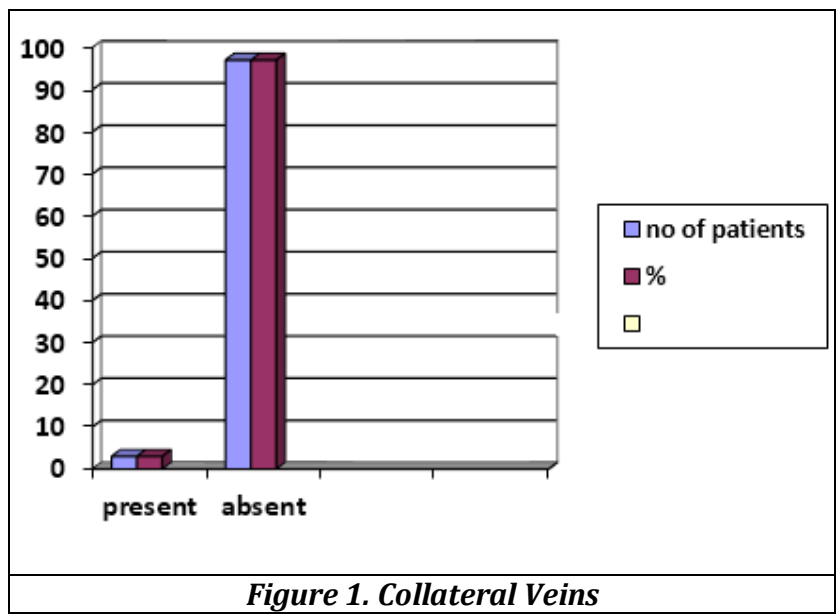

All 100 patients were examined by colour flow mapping. In our study, we found that $100 \%$ of patients had absent resting flow and no evidence of colour flow was seen on distal augmentation above the site of thrombosis.

The patients were distributed on the basis of Phasic variation and reflux on Valsalva findings: In our study we found that distal to the site of thrombosis, there was no evidence of Phasic variation seen. In our study we found that distal to the site of thrombosis, there was no evidence of reflux on Valsalva seen.

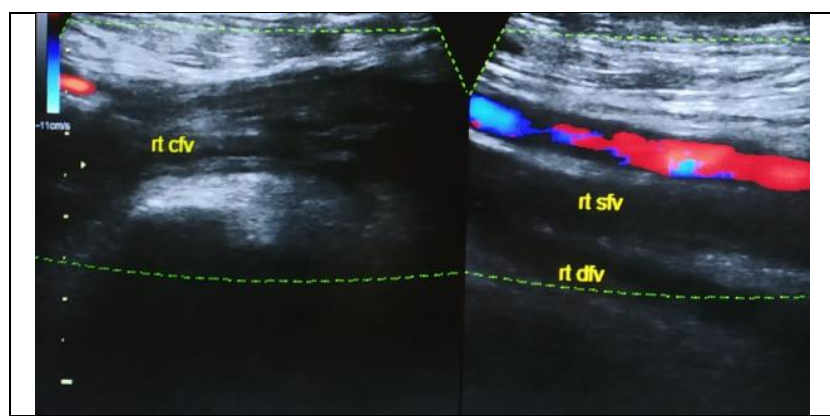

Figure 2. Absent colour flow signal in lumen of right common femoral vein, superficial femoral vein and deep femoral vein. Note-Diameter of right superficial femoral vein is more than right superficial femoral artery

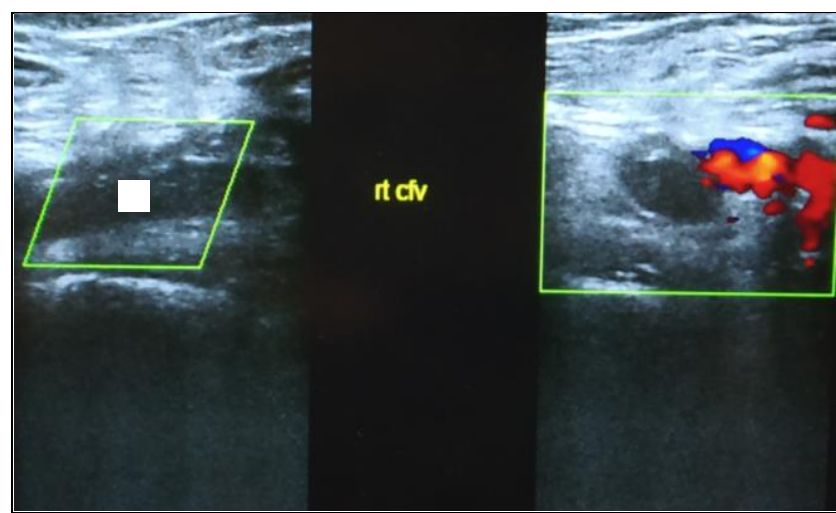

Figure 3. Absent colour flow signal in lumen of right common femoral vein. Note-Diameter of right common femoral vein is more than right superficial femoral artery 


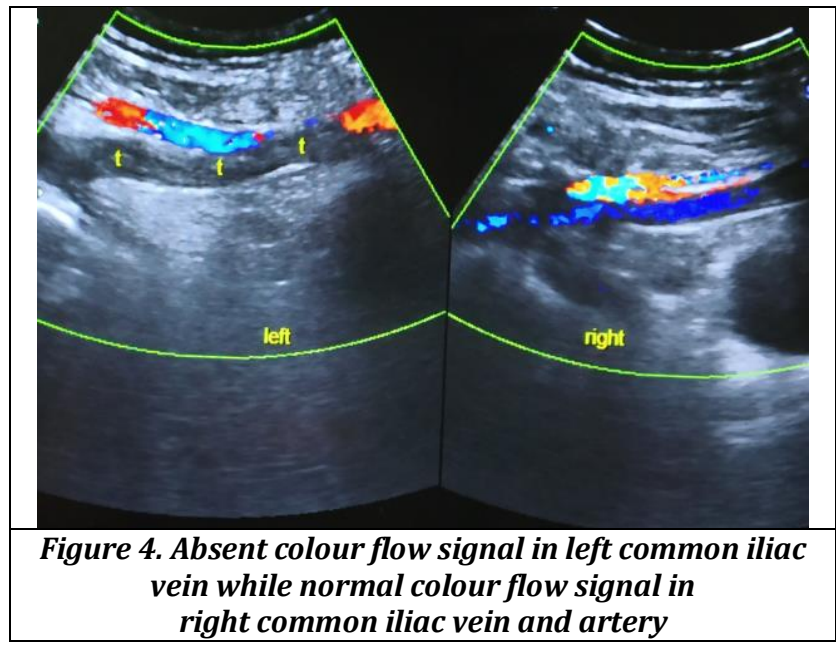

\section{DISCUSSION}

Majority of patients (60\%) are of age group of $40 \mathrm{yrs}$ and above because they are more prone to diseases like stroke, malignancy and road traffic accidents.

In our study 51 patients were males and 49 patients were females with male to female ration of 1.02:1 and comparing this with published literature by Wells et al[1] in which ratio of 1.5:1, we find no major difference in males and females as far as the incidence of acute DVT is concerned.

In our study, the findings relative to location and extent of thrombus of involvement is as follows-

1. The right leg was involved in $41 \%$, the left leg was involved in $49 \%$ and bilateral involvement was in $10 \%$.

2. The veins most frequently involved in acute DVT were as follow:

a. SFV: $84 \%$, PV: $74 \%$, CFV: $64 \%$, PTV: $44 \%$, ATV: 23\%, DFV: $24 \%$, EIV: 14\%, CIV: 4\%, PeV: $8 \%$, IVC: $6 \%$, GSV: $10 \%$ and SSV: $4 \%$.

3. In our study, isolated involvement of SFV in acute DVT was seen in only $3 \%$ of cases.

4. In symptomatic acute DVT, proximal involvement was seen in $94 \%$ patients, and in only $6 \%$ of patients there was isolated calf vein thrombosis. Bilateral involvement was more commonly seen in proximal DVT.

According to S. C. Rose et al,[2] Among symptomatic patients with acute DVT, 26 of $34(76 \%)$ patients had an above-knee thrombus and only eight of 34 (24\%) patients had a thrombus isolated to the calf. In comparison, only three of $25(12 \%)$ asymptomatic patients with DVT had an aboveknee thrombus and 22 of $25(88 \%)$ patients had a thrombus isolated to the calf veins (most involving only one venous segment).

According to Maki et al[3] Of 2704 lower extremities studied with duplex sonography, acute deep venous thrombosis was isolated to the superficial femoral vein in $60(22.3 \%)$. The remaining 209 cases (77.7\%) showed thrombus that extended into the common femoral or popliteal veins (or both). However, in our study the percentage of patients with isolated superficial femoral vein thrombosis is only $3 \%$.

In our study we found that on B-mode study, anechoic thrombus was present in $55 \%$ of patients and heterogenous
(Anechoic to isoechoic) thrombus was seen in $45 \%$ of patients. On compression ultrasound loss of compressibility was seen in $98 \%$ of patients whereas in only $2 \%$ of patients the dilated vein was compressible. On colour Doppler study resting flow was absent in $100 \%$ of patients. Hence, from our study we found that colour Doppler facilitated a better sensitivity for diagnosis of acute DVT.

\section{Comparing this with the earlier studies}

Baxter et al,[4] the sensitivity and specificity of colour Doppler ultrasound for diagnosis proximal deep venous thrombosis in symptomatic patients was $100 \%$ and $100 \%$ respectively.

Mattos et al,[5] the sensitivity and specificity of colour Doppler ultrasound for diagnosis proximal deep venous thrombosis in symptomatic patients was $100 \%$ and $98 \%$ respectively.

Elias et al[6] the sensitivity and specificity of B-mode ultrasound for diagnosis proximal deep venous thrombosis in symptomatic patients was $100 \%$ and $98 \%$ respectively.

Quintawallaet al,[7] the sensitivity and specificity of Bmode ultrasound for diagnosis proximal deep venous thrombosis in symptomatic patients was $97 \%$ and $98 \%$ respectively.

In our study, vein lumen diameter was increased in $97 \%$ of patients, decreased in $2 \%$ of patients and normal in $1 \%$ of patients.

According to Barbara S Hertzberg et al,[8] veins with acute DVT were larger than normal veins. Likewise, veins with chronic DVT were smaller than normal veins.

\section{Reported Accuracy}

A study was conducted by Steven C Rose et al[9]in University of Salt Medical centre, Salt lake city in which 69 consecutive patients were studied who underwent both venography and colour Doppler flow imaging. Colour Doppler flow imaging results for detection of deep vein thrombosis above the knee (both iliac and femoropopliteal venous segments) were as follows:

Sensitivity $96 \%$, specificity $100 \%$, positive predictive value $100 \%$, negative predictive value $98 \%$, accuracy $99 \%$.

Detection of deep vein thrombosis between the inguinal ligament and knee (Common femoral, superficial femoral, deep femoral and popliteal veins) sensitivity $92 \%$, specificity $100 \%$, positive predictive value $100 \%$, negative predictive value $96 \%$, accuracy $97 \%$.

For detection of thrombus of thrombus in the infrapopliteal deep veins (Tibioperoneal trunk and trunks and branches of the posterior tibial and peroneal veins) results were as follows: sensitivity $73 \%$, specificity $86 \%$, positive predictive value $79 \%$, negative predictive value $83 \%$, and accuracy $81 \%$.

In a study done by Rose et al[2] 1994, in which Doppler ultrasound of 75 patients was done for diagnosis of symptomatic deep venous thromboses following results were obtained:

In evaluation of iliac and femoropopliteal veins, sensitivity $96 \%$ and specificity $100 \%$ was seen. Doppler ultrasound of femoropopliteal veins only showed $92 \%$ sensitivity and $100 \%$ specificity.

In study of calf veins only $73 \%$ sensitivity and $86 \%$ specificity were seen. Adequate calf vein studies in 45 
patients by Rose et al showed 95\% sensitivity and 100\% specificity.

In another study, by Baxter et al,[4] 40 symptomatic patients were studies. Femoropopliteal vein study showed $100 \%$ sensitivity and $100 \%$ specificity, while calf veins Doppler ultrasound results were: 95\% sensitivity and 100\% specificity.

The diagnostic sensitivity of calf vein imaging is a point of controversy although values ranging from $88 \%$ to $95 \%$ have been reported.

In our study we found common etiological factors causing acute DVT in decreasing order of frequency include post traumatic, post-surgical, purpureal, chronic medical diseases (CVA, post myocardial infarction, nephritic syndrome) \& malignancy, insect bite. In our study $50 \%$ of total causes were secondary to trauma, recent surgery \& chronic medical diseases. On comparing with study done by Alberto Cogo et al.[10] Approximately 50\% of cases of DVT were considered to be secondary to a major risk factor (Immobilization, trauma, and/or recent surgery). Among additional risk factors, only increased age (Over 60 years), male gender, malignant neoplasm, heart failure, systemic lupus erythematosus, and arteriopathy were independently associated with the risk of acute DVT.

\section{CONCLUSION}

In 100 patients of acute DVT using B- Mode Ultrasonography \& Colour Doppler study, we made the following obsevations in symptomatic patients of acute DVT-

In about $94 \%$ of symptomatic patients, thrombus was seen involving the veins above knee that is the femoropopliteal segment.

In symptomatic patients isolated involvement of superficial femoral vein found only in 3\% patients.

While isolated calf vein involvement was seen in $6 \%$ of patients. Bilateral involvement was seen more commonly in proximal DVT (Ilio-femoral).

Probe compressibility was absent in B-Mode ultrasonography in $98 \%$ patients who had deep vein thrombosis \& it can be concluded that it is the more specific criterion to diagnose DVT on B-Mode sonography.

On colour Doppler study, resting colour flow was absent in $100 \%$ patients \& it can be concluded that colour Doppler is more sensitive $\&$ also facilitates localization of vessel.

We were able to identify the morphology of thrombus according to echogenicity whether it is anechoic or hypoechoic as a criterion to determine the chronicity of thrombus which correlated with the duration of complaints of the patient.

Lumen diameter was found to be increased in acute DVT usually, more than twice the corresponding artery \& on probe compression there was absent probe compressibility thus simplifying the technique of diagnosing acute DVT.

In our study, $>50 \%$ patients had DVT secondary to trauma, recent surgery \& chronic medical diseases.

Thus, ultrasonography \& colour Doppler is a better predictor of acute DVT because it is more sensitive \& specific, non-invasive, painless, widely available, easy to use, and less expensive \& has no ionizing radiations.

\section{ACKNOWLEDGMENT}

We acknowledge Dr. Rajendra Gupta for guiding us in every step of research work. We also acknowledge Dr. Mukesh Patidar, Dr. Parul Gupta, Dr. Neeraj Jain, Dr. Namrata Kotangle, Dr. Chandra Pratap Singh, Dr. Bhagyashree Patil, Dr. Agamya Saxena, Dr. Namita Kamath and Dr. Manisha for her assistance with the radiologic findings and Mr. Indal Singh for his assistance in statistical analysis. We would also like to thank Mr. Shri Jalim Singh, Smt. Pushpa Singh, Smt. Pooja Singh, Mr. Rahul Shrivastava, Mr. Akhilesh Pathak, Mr. Anuj Singh Senger and Dr. Ravikant Gupta for their support and technical help during study.

\section{REFERENCES}

[1] Wells PS, Hirsh J, Anderson DR, et al. Accuracy of clinical assessment of deep-vein thrombosis. Lancet 1995;345(8961):1326-30.

[2] Rose SC, Zwiebel WJ, Miller FJ. Distribution of acute lower extremity deep venous thrombosis in symptomatic and asymptomatic patients: imaging implications. J Ultrasound Med 1994;13(4):243-50.

[3] Maki DD, Kumar N, Nguyen B, et al. Distribution of thrombi in acute lower extremity deep venous thrombosis: implications for sonography and CT and MR venography. Am J Roentgenol 2000;175(5):1299301.

[4] Baxter GM, McKechnie S, Duffy P. Colour Doppler ultrasound in deep venous thrombosis: a comparison with venography. Clin Radiol 1990;42(1):32-6.

[5] Mattos MA, Londrey GL, Leutz DW, et al. Color-flow duplex scanning for the surveillance and diagnosis of acute deep venous thrombosis. J Vasc Surg 1992;15(2):366-76.

[6] Elias A, Le Corff G, Bouvier JL, et al. Value of real time B mode ultrasound imaging in the diagnosis of deep vein thrombosis of the lower limbs. Int Angiol 1987;6(2):175-82.

[7] Quintavalla R, Larini P, Miselli A, et al. Duplex ultrasound diagnosis of symptomatic proximal deep vein thrombosis of lower limbs. Eur J Radiol 1992;15(1):32-6.

[8] Hertzberg BS, Kliewer MA, DeLong DM, et al. Sonographic assessment of lower limb vein diameters: implications for the diagnosis and characterization of deep venous thrombosis. American Journal of Roentgenology 1997;168(5):1253-7.

[9] Rose SC, Zwiebel WJ, Murdock LE, et al. Insensitivity of color Doppler flow imaging for detection of acute calf deep venous thrombosis in asymptomatic postoperative patients. Journal of Vascular and Interventional Radiology 1993;4(1):111-7.

[10] Cogo A, Bernardi E, Prandoni P, et al. Acquired risk factors for deep-vein thrombosis in symptomatic outpatients. Arch Intern Med 1994;154(2):164-8. 